\title{
The Effectiveness of Group Therapy in Supporting Adoptive Families
}

\author{
Kristen DeGree, Shanika Lavi Wilson*, Christopher Solomon \\ North Carolina Central University \\ *Corresponding author: Shanika Lavi Wilson, North Carolina Central University
}

Received date: April 01, 2021; Accepted Date: May 08 2021; Published Date: May 14, 2021.

Citation: Kristen DeGree, Shanika Lavi Wilson, Christopher Solomon. The Effectiveness of Group Therapy in Supporting Adoptive Families, J. Psychology and Mental Health Care, 5(2). Doi: 10.31579/2637-8892/122

Copyright: () 2021 Shanika Lavi Wilson, This is an open-access article distributed under the terms of the Creative Commons Attribution License, which permits unrestricted use, distribution, and reproduction in any medium provided the original author and source are credited.

\begin{abstract}
Adopted children and their families have unique needs compared to non-adoptive families. Adoption research seeks to understand these unique needs and contribute to a growing field of adoption competent therapy. The purpose of this paper is to understand how adoption competent group therapy can benefit adoptive families, and provides analysis on secondary data collected in 2019 from pre and post measures for a weekly support group from post-adoption program. The research included 8 participants who were adoptive parents of adolescent girls, and included quantitative and qualitative data about how parents and their children responded to the program. The questions covered a variety of topics regarding the child, adoptive parents, and the parent-child relationship as a while. The results showed that from a quantitative standpoint, minimal improvements were made after the group, but the qualitative data showed that the group provided numerous benefits for adoptive parents.
\end{abstract}

Keywords: adoption; adoptive families; group therapy; adoption competency

Current data reveals that in the United States around 7 million people are adopted, and around 140,000 children are adopted each year (Adoption Facts, 2013). Nearly 100 million people have a family connection with adoption in the United States, whether someone is adopted within their family, or they were adopted themselves (Adoption Facts, 2013). Moreover, 6 in 10 Americans either have a family connection to adoption, have close friends who are adopted, or have placed a child for adoption (Adoption Facts, 2013). While historically, adoptions were conducted privately, it is apparent that a majority of people in the United States are affected by adoption in different ways (Adoption Facts, 2013). Research on the effects of adoption for children and adults has evolved over the last 50 years (Palacios \& Brodzinsky, 2010). It is only recently that adoption competency has been introduced as an important quality in treatment (Atkinson, Gonet, Freundlich, \& Riley, 2013). This paper seeks to understand the effectiveness of adoption competent therapy, and identify ways it can help families.

\section{Literature Review}

\section{The Unique Needs have Adopted Children}

Palacios \& Brodzinsky (2010) consider one of the early trends in adoption-related research to be whether adopted children have more mental health problems than their non-adopted peers. For example, research has found that adopted children may have a higher need for mental health treatment than non-adopted children, and adopted children are 2.5-6 times more likely to be represented in mental health clinics and agencies, and 2-3.7 times more likely to seek clinical treatment (Keyes,
Sharma, Elkins, Iacono, \& McGue, 2008). Other studies have found the adopted children who are in residential treatment or inpatient psychiatric centers to be at a higher rate, from 9-21\% (Brodzinsky, Smith, \& Brodzinsky, 1998). However, other research explains these higher rates as due to adoptive parents being more willing to seek treatment for their child, and more aware of mental health issues (Peters, Atkins, \& McKernan McKay, 1999; Miller, Fan, Grotevant, Christenson, Coyle, et al., 2000). Other studies have refuted the higher rates of adoptive children and mental health issues, including a 2011 study which compared adopted adolescents with non-adopted teens in the context of externalizing behaviors such as aggression, hostility, and opposition. The study found that the presence of problematic behaviors were similar in both groups, showing that adoption status alone could not determine whether a child would be predisposed to externalizing behaviors (Koh \& Rueter, 2011).

However, research has found that adopted children have unique needs compared to children who have not been separated from their birth families. Research suggests that childrens' needs in understanding their adoption may be different based on their development (Brodzinsky, 2011). Adopted children may have feelings of loss and grief attached to their adoption, and children process this loss differently at each stage of their development (Brodzinsky, 2011). Specifically, as adoptees process the separation from their birth parents, less attention is given to those grieving for other caregivers they may have lost, whether in foster care or an orphanage. Additionally, adopted children may feel stigma or loss of social standing with their peers, if those around them have negative feelings about adoption (Brodzinsky, 2011). In international adoptions, or children who were adopted to parents of a different race, children may 
feel cut off from the race, ethnicity, and culture of their birth family (Brodzinsky, 2011; Anderson, Rueter, \& Lee, 2015).

\section{The Need for Adoption Competent Therapy}

Adoption affects an increasing number of people in the United States, and research has shown that adoptees have unique needs and treatment goals in therapy. The growing number of people "touched by adoption" shows a significant need for adoption competent therapists.

Participants in a 2013 study defined adoption competent therapy as understanding that there are fundamental differences between those who were adopted and those who were not (Atkinson, et al., 2013). A 2013 study found that only $14 \%$ of adult adoptees believed that their therapist was competent in engaging their adoption story, and $24 \%$ of participants felt that mental health professionals had no understanding of adoption language, and were not equipped to engage adoptees about attachment, loss, trauma, or adoption (Atkinson, et. al, 2013). There has not been an empirical study that determines the adoption competence of therapists (Baden, Kitchen, Mazza, Harrington, \& White, 2017). Baden et. al conducted an exploratory study among adult adoptees that found adoption competence to be the primary need for participants seeking a therapist (2017). Moreover, participants linked the therapists' willingness to engage with the clients' adoption with how satisfied they were with their treatment (Baden, Kitchen, Mazza, Harrington, \& White, 2017).

\section{Adoption-Competent Therapy for Adoptive Parents}

Whether a child suffers from mental health issues or not, there are a number of influences that may strain relationships between the child and adoptive parent. Some adoptive parents do not always feel prepared to handle their child's emotional and psychological challenges (O'Dell, McCall, \& Groak, 2015). An 2018 exploratory study of adoptive parents summarized some of the most urgent concerns for parents, including $28 \%$ of participants reporting attachment issues with their child and 32\% expressing a need for psychoeducation geared towards adoption (Wade

\& Alewine, 2018). The study also found indications of caregiver strain, as $31 \%$ of participants reported a need for therapy, and $23 \%$ reported feeling isolated due to their family challenges (Wade \& Alewine, 2018). Adoptive parents struggle with how to share adoption information with their child, as well as how to help their child make meaning of their adoption journey in a functional, healthy way (Palacios \& Brodzinsky, 2010). This research suggests that adoptive parents need a support system just like parents who have built their family through biological means, but their needs may be more unique. A 2017 report from the United Kingdom of adoptive parents who participated in a qualitative study found that $93 \%$ of participants feel that society doesn't understand adoptive families (Weistra \& Luke, 2017). Adoptive parents often feel misunderstood in the context of families that formed biologically, either feeling that their family connection is less "real," or that they are heroic, leading to inflated expectations about how they should be perfect parents. The presence of a non-judgmental group to share frustrations was noted as helpful, showing the need to both normalize their struggles and lessen the feeling of stigma around adoption (Weistra \& Luke, 2017).

Whether adopted children are predisposed to mental health issues or not, Roszia and Maxon (2019) write that it is important to acknowledge that a loss has occurred for those involved, including the child, biological parent, and adoptive parent. For adoption to occur, the child has been separated from their birth parents for any number of complex reasons, and is a loss which both the child and biological parents mourn in some way. For adoption to occur, sometimes adoptive parents have also suffered a loss which led them to build their family through nonbiological means, whether due to infertility or the loss of a child. Some parents may grieve the "fantasy child" that they thought they were adopting after they begin to encounter conflicts in their relationship with their child (Roszia \& Maxon, 2019).

There are a number of new methods to help parents and children cope with the stress of grief of adoption. A growing number of studies suggest that building a narrative or family history can be helpful for adopted children to make meaning from their adoption (Kranstuber \& Kellas, 2011; Stokes \& Poulsen, 2014). Group therapy can help parents meet other adoptive parents, and help with the isolation that some adoptive parents feel, as society tends to place emphasis on biological births over chosen families (Weistra \& Luke, 2017). A group setting can also help parents share adoption specific resources or assistance that would be otherwise difficult to find (Wade \& Alewine, 2018). A 2008 pilot study in Kentucky found that an adoptive parent-led support group helped stabilize families, and offered a safe space for the group members to share frustrations (Bryan, Flaherty, \& Saunders, 2010). As an added bonus, support groups that include children can help normalize adoption when children meet fellow adoptees or children in foster care (Maldonado, 2009).

\section{Program Description}

Learning and Empowerment for Adoptive Families (LEAF) is a group therapy program created by Post Adoption Support Services at Center for Child and Family Health (CCFH) in Durham, North Carolina (Workshops, n.d.). LEAF was adapted in part by resources from the Center for Adoption Support and Education (CASE) in Maryland (K. Murray, personal communication, January 9, 2020). The Post Adoption program operates through a grant from the North Carolina Department of Health and Human Services (NCDHHS), and LEAF is one of the free services that Post Adoption provides for adoptive families in their regions (Workshops, n.d.).

The group therapy sessions for LEAF includes 10 weekly programs with a separate curriculum for parents and children, with weekly calls from clinicians regarding how both parents and their child are responding to the group (Workshops, n.d.). LEAF is led by clinicians within the Post Adoption program, as well as parent facilitators, who are fellow adoptive parents who have participated in previous sessions of LEAF. The content introduces adoption language for both parents and children to discuss their adoption, and supports parents on processing their child's feelings around their adoption. Every week, LEAF provides a new coping skill to help parents and children stay calm, allowing for parents to connect with their child during times of stress. Additionally, LEAF provides group activities to help children process their adoption story with fellow adopted children (Workshops, n.d.).

Although LEAF is not an evidence-based treatment, Dr. Kate Murray (personal communication, January 9, 2020), the co-director of Post Adoption Support Services at CCFH, states that the curriculum utilizes evidence-based treatments including the Training for Adoption Competency from CASE. LEAF also includes individual activities that are evidence-based, such as feelings identification from Cognitive Behavioral Therapy, adoption narrative building from Trauma-Focused Cognitive Behavioral Therapy, emotional regulation from Dialectical Behavioral Therapy and Attachment, Regulation, and Competency (K. Murray, personal communication, January 9, 2020). As LEAF is one of the post adoption services funded by NCDHHS, some outcomes outlined by the grant are to prevent the dissolution of families, provide educational opportunities for parents, and perform therapy services for adoptive families (K. Murray, personal communication, January 9, 2020). However, CCFH has additional outcomes including giving adoptive parents and children coping tools for dealing with difficult feelings around adoption, facilitating the parents' and children's conversations about adoption, helping children learn adoption language to talk about their adoption, and providing social opportunities for adoptive families 
(Workshops, n.d.). The curriculum is still being adapted based on the pre and post measures and individual feedback completed by participating parents.

\section{Theoretical Framework}

Shared fate theory, introduced by H. David Kirk (1964), can be a useful tool in understanding the unique needs of adoptive families, as it recognizes the fundamental differences experienced by adoptive families as part of a "shared fate" between the adoptive parent and their child (Kirk, 1964). After collecting interviews with a wide variety of adoptive parents in different geographic areas, Kirk determined that adoptive parents may suffer a "role handicap," in that they may feel differently than parents who built their family through biological means (1964). Originally published in 1964, Shared Fate was written when adoption was taboo in part because it suggested that adoptive parents were infertile, or had reproductive issues that didn't allow them to conceive. Kirk, an adoptive parent himself, felt that adoptive parents coped with this role by either rejecting their differences, or acknowledging their differences. The "rejection-of-difference," meant a denial of any difference between adoptive families and biological families (Kirk, 1964). Kirk found that those who acknowledged that their shared fate specifically that their families were built through different means had better communication with their children and a healthier level of family functioning (1964). In Shared Fate, Kirk is also an early advocate of open adoptions in a time when most adoptions were closed and secret (1964).

\section{Methodology}

This is a secondary data analysis of research, meaning that the data has already been collected by a separate agency, and includes data from pre and post measures. The measures include quantitative and qualitative date completed by eleven parents who participated in the summer 2019 LEAF program. The session was held at a therapeutic partner organization in North Carolina. LEAF was held by request of the organization for nine at-risk adolescent girls and current clients of the therapeutic partner organization. The participants included the girls and their adoptive parents, and was led by four clinicians and one parent facilitator who had participated in a previous LEAF program. The goals of LEAF were to help children gain confidence in expressing themselves and handling their

\begin{tabular}{|l|l|l|}
\hline Parent & \multicolumn{1}{|c|}{$\begin{array}{c}\text { Pre-Workshop } \\
\text { (SD) }\end{array}$} & $\begin{array}{c}\text { Post-Workshop } \\
\text { M (SD) }\end{array}$ \\
\hline $\begin{array}{l}\text { Understands what their child is } \\
\text { thinking and feeling }\end{array}$ & $2.88(1.42)$ & $3.13(1.79)$ \\
\hline $\begin{array}{l}\text { Is confident they can respond } \\
\text { effectively when child is upset }\end{array}$ & $2.88(1.59)$ & $3.75(1.5)$ \\
\hline Knows other adoptive parents they & $4.56(1.13)$ & $4.56(1.01)$ \\
\hline can talk to when needed & & \\
\hline
\end{tabular}

\begin{tabular}{|l|l|l|}
\hline $\begin{array}{l}\text { Is aware of how their own history } \\
\text { may impact parent-child relationship }\end{array}$ & $3.11(1.62)$ & $4(1.32)$ \\
\hline $\begin{array}{l}\text { Can tell what child is really feeling, } \\
\text { despite behavior }\end{array}$ & $3.78(1.2)$ & $4.11(1.27)$ \\
\hline
\end{tabular}

Note. $\mathrm{M}=$ Mean; $\mathrm{SD}=$ Standard Deviation.

\section{Table 1: Areas of Improvement within Parent Domain}

For the child domain, parents reported their perceptions of their child's behavior using Likert items that rated 1 for Strongly Agree, 2 for Disagree, 3 for Slightly Disagree, 4 for Slightly Agree, 5 for Agree and 6 feelings about their adoption, and to help parents navigate their child's feelings about their adoption, learn coping skills for parenting, and improve their connection with their child. As an added outcome, being around other adoptive families can help normalize adoption for both children and parents (Workshops, n.d.).

The quantitative data was collected on paper surveys that were distributed before and after the ten week workshop series, and included multiple choice questions for demographics, and utilized a Likert scale for survey questions. Likert scale questions engaged parents on their comfort level in dealing with their child's emotions, talking with their child about their adoption, handling difficult feelings around their child's adoption, and how their child's feelings triggered their own emotions. Additionally, within the post-workshop survey, qualitative data was collected in the form of handwritten feedback from parents. The pre and post measures are used to refine and improve the LEAF curriculum, fulfill grant information, as well as improve program outreach (K. Murray, personal communication, January 9, 2020). Therefore, this secondary analysis selected data that related to the research question. The results that were analyzed included responses that measured the parent's emotional connection with their child, willingness to engage in conversations about adoption, and their child's emotional well being from the parents' perspective.

\section{Results}

\section{Quantitative Data}

While the child participants were all adolescent females, the parent demographic was racially diverse, with a range of educational and economic backgrounds. While LEAF ran with a group of eleven participants, three measures were discarded because the respondents did not complete all of the questions. The secondary analysis organized categories based on the parent domain, child domain, and parent-child domain. For the parent domain, parents responded to a series of questions using Likert items that rated 1 for Strongly Agree, 2 for Disagree, 3 for Slightly Disagree, 4 for Slightly Agree, 5 for Agree and 6 for Strongly Agree. A paired-samples t-test was conducted to compare the average scores from the pre and post-workshop conditions. In terms of probability, there was not a significant difference in the scores for the pre and postworkshop conditions $(\mathrm{p}>0.50)$.

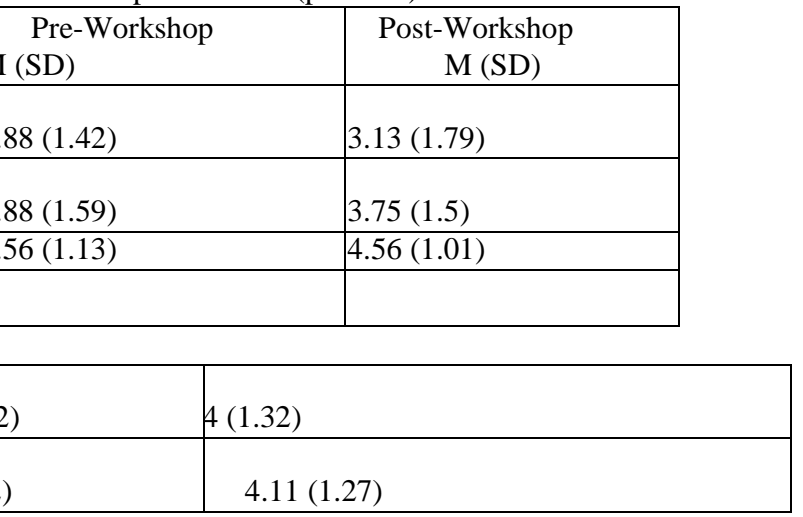

for Strongly Agree. A paired-samples t-test was conducted to compare the average scores from the pre and post-workshop conditions. In terms of probability, there was not a significant difference in the scores for the pre and post-workshop conditions $(\mathrm{p}>0.50)$. 


\begin{tabular}{|l|l|l|}
\hline Child & \multicolumn{1}{|c|}{$\begin{array}{c}\text { Pre-Workshop } \\
\text { M (SD) }\end{array}$} & $\begin{array}{l}\text { Post-Workshop } \\
\text { M (SD) }\end{array}$ \\
\hline $\begin{array}{l}\text { Regularly shares feelings about } \\
\text { adoption }\end{array}$ & $3(1.66)$ & $3.5(1.9)$ \\
\hline Knows coping skills & $2.63(1.8)$ & $3.25(1.62)$ \\
\hline Uses coping skills & $2(1.64)$ & $2.5(1.56)$ \\
\hline Feels pride regarding their adoption & $2(1.3)$ & $2.36(1.83)$ \\
\hline $\begin{array}{l}\text { Shows confidence in responding to } \\
\text { questions about adoption }\end{array}$ & $3.13(1.78)$ & $2.88(1.81)$ \\
\hline
\end{tabular}

Note. $\mathrm{M}=$ Mean $; \mathrm{SD}=$ Standard Deviation.

Table 2: Areas of Improvement within the Child Domain

For the parent-child relationship domain, parents reported their comfort with the topics using Likert items that rated 1 for Extremely Uncomfortable, 2 for Moderately Uncomfortable, 3 for Slightly Uncomfortable, 4 for Slightly Comfortable, 5 for Moderately
Comfortable and 6 for Extremely Comfortable. A paired-samples t-test was conducted to compare the average scores from the pre and postworkshop conditions. In terms of probability, there was not a significant difference in the scores for the pre and post-workshop conditions ( $p$ > $0.50)$.

\begin{tabular}{|l|l|c|}
\hline Parent-child relationship & \multicolumn{1}{|c|}{$\begin{array}{c}\text { Pre-Workshop } \\
\text { M (SD) }\end{array}$} & $\begin{array}{r}\text { Post-Workshop } \\
\text { M (SD) }\end{array}$ \\
\hline $\begin{array}{l}\text { Dealing with child's adoption related } \\
\text { loss }\end{array}$ & $3.56(1.51)$ & $4.11(1.76)$ \\
\hline Talking about child's birth culture & $5.33(0.71)$ & $4.88(1.54)$ \\
\hline $\begin{array}{l}\text { Dealing with child's difficult } \\
\text { emotions }\end{array}$ & $2.56(0.88)$ & $3.67(1.58)$ \\
\hline $\begin{array}{l}\text { Dealing with parent's difficult } \\
\text { emotions }\end{array}$ & $3.00(1.5)$ & $3.44(1.51)$ \\
\hline $\begin{array}{l}\text { Answering questions about birth } \\
\text { family }\end{array}$ & $4.00(1.22)$ & $4.22(1.48)$ \\
\hline
\end{tabular}

Note. $\mathrm{M}=$ Mean $; \mathrm{SD}=$ Standard Deviation.

Table 3: Areas of Improvement within the Parent-Child Relationship

\section{Qualitative Results}

Within the feedback portion of the post-workshop measures, parents were able to write individual responses about how they felt the workshop had impacted them. At least two parents wrote that the workshop helped them better understand adoption feelings and sense of loss for their child. A majority of parents (6) felt that learning coping skills like deep breathing and tools to help connect with their child was the most helpful. One parent shared that it changed their perspective and made them more tolerant of their child, and was not as quick to punish them for their behavior. Additionally, a majority of parents (5) felt that talking with other parents with similar issues with their own children was the most helpful part of the workshop.

Parents were also asked to provide feedback on what was most helpful for their children. Parents wrote that it helped their child consider their adoption story, and work through adoption feelings. Half of parents found that for their child, getting to know other adopted kids and share their stories was the most impactful part of the group. Additionally, three parents wrote that they noticed their child became more comfortable sharing her thoughts and feelings about being adopted with her parents during the workshop. Regarding areas of improvement for the workshop, multiple parents expressed a desire for more time to talk with other parents, and for them, the group sharing was the most helpful part of the workshop.

\section{Conclusion}

The purposes of this study were to better understand the effectiveness of adoption competent therapy, and identify ways it can help families. There was not a significant statistical difference in the scores for the pre and post-workshop conditions for any of the measures, so this study was unable to verify whether LEAF had the expected impact on families. However, this could be due to the small sample size (8), and the fact that each parent had varied levels of improvement within the different domains. For example, in response to the statement, "I regularly think about how my own history impacts how I respond to my child," one parent responded as strongly disagreeing to the statement before the workshop and strongly agreeing after the workshop. A second parent moved from disagreeing before the workshop to slightly agreeing after the workshop. For at least two parents, this portion of the workshop had a positive impact on their awareness of how their own history impacts the ways they engage with their child. Additionally, decreased numbers may indicate that the workshop has drawn attention to an area where parents have previously not considered an issue. For the same question, one parent shifted from being in agreement before the workshop down to being in slight 
agreement after the workshop. This could lead one to speculate that the impact of the group may have caused the parent to interpret the question differently after the workshop. Similar results were found with the question, "How comfortable do you feel talking about your child's birth culture, (e.g. race, ethnicity, country of origin)?" One parent reported being extremely comfortable before the workshop to being extremely uncomfortable talking about their child's birth culture after the workshop. Another parent reported feeling extremely comfortable prior to the workshop to moderately comfortable after the workshop. One could speculate that some parents may have gained an understanding of the significance of their adoptive child's birth culture and now felt differently about the question. Overall, more research needs to be completed in this area to determine whether the workshop has more widespread impacts for the participants.

While the quantitative data may be inconclusive, the qualitative results shows that on a practical level, the group had a positive impact for families. For example, a majority of the parents (6) wrote that learning coping skills to help them connect with their child was helpful, as well as being able to talk with other parents. Half of parents found that letting their child engage with other adopted kids was the most impactful part of the group, and nearly half of participants noticed their child became more comfortable sharing her adoption story with them.

The goals of the LEAF are to give adoptive parents and children coping skills for to process difficult feelings around adoption, engage parents' and children's conversations about adoption, help children develop adoption language to talk about their adoption, and provide a social outlet for adoptive families (Workshops, n.d.). Based on the individual feedback from parents, one canconclude that the workshop fulfilled its outcomes on a practical scale.

One of the main limitations of the study is that the measures were not approved by the Institutional Review Board. Another limitation is the small size of the group, which makes it difficult to determine whether the group had a widespread impact on its participants. With this small group, a few parents with varied experience were enough to skew the data towards reflecting less of an impact than is suggested by the qualitative data. Lastly, a limitation could also be that parents are reporting on their perspective of their child, which might not accurately represent the impact of the group for the child. In the future, a self reporting measure for children might also be helpful to understand the impact.

The implications for social work practice can be understood through the preexisting research, as well as the responses given by the participants that shows the need for more adoption competent group therapy programs. It is clear that adoptive parents feel the need for more social space with other adoptive parents to share the challenges, as well as coping skills and resources unique to adoptive families. It is also clear that the adoptive parents found the group impactful for their children, both in becoming comfortable talking about their adoption story, as well as sharing space with other adopted children. As many social workers work with adoptive families, it would be beneficial to introduce more training programs for social workers to gain adoption competency, as well as increase the availability of group workshops like LEAF for families in the future.

\section{References}

1. Adoption Facts. (2013). Adoption research.
Atkinson, A. J., Gonet, P. A., Freundlich, M., and Riley, D. B. (2013). Adoption competent clinical practice: Defining its meaning and development. Adoption Quarterly, 16(3), 156-174.

3. Baden, A. L., Kitchen, A., Mazza, J. R., Harrington, E. S., and White, E. E. (2017). Addressing adoption in counseling: A study of adult adoptees' counseling satisfaction. Families in Society: The Journal of Contemporary Social Services. 98(3), 209-216.

4. Brodzonsky, D. (2011). Children's understanding of adoption: Developmental and clinical implications. Professional Psychology: Research and Practice, 42(2), 200-207.

5. Brodzinsky, D. M., Smith, D. W., \& Brodzinsky, A. B. (1998). Children's adjustment to adoption: Developmental and clinical issues. Thousand Oaks, CA: Sage.

6. Bryan, V., Flaherty, C., and Saunders, C. (2010). Supporting adoptive families: Participant perceptions of a statewide peer mentoring and support program. Journal of Public Child

7. Keyes, M. A., Sharma, A., Elkins, I. J., Iacono, W. G., and McGue, M. (2008). Mental health of Pediatric Adolescent Medicine, 5 ,

8. Kirk, H. D. (1964). Shared fate: A theory of adoption and mental health. London, UK: The Free Press of Glencoe

9. Koh, B., \& Reuter, M. A. (2011) Contributions of parentadolescent negative emotionality, adolescent conflict, and adoption status to adolescent externalizing behaviors. Journal of Clinical Child and Adolescent Psychology, 40(6). 825-836.

10. Kranstuber, H. \& Kellas, J. K. (2014) "Instead of growing under her heart, I grew in it": The relationship between adoption entrance narratives and adoptees' self-concept. Communication Quarterly, 59(2), 179-199.

11. Maldonado, J. (2009). Using group therapy to improve the wellbeing of children in foster care. (Master's thesis, The College at Brockport: State University of New York, United States).

12. Miller, B. C., Fan, X., Grotevant, H. D., Christenson, M., Coyle, D., et al. (2000). Adopted adolescents' overrepresentation in mental health counseling: Adoptees problems or parents lower threshold for referral? Journal of the American Academy of Child and

13. O'Dell, K. E., McCall, R. B., and Groak, C. J. (2015). Supporting families through the international special needs adoption process. Children and Youth Services Review, 59,

14. Palacios, J., and Brodzinsky, D. (2010). Adoption research: Trends, topics, outcomes. International special needs adoption process. Children and Youth Services Review, 59,

15. Peters, B. R., Atkins, M. S., and McKernan McKay, M. (1999). Adopted children's behavior problems: A review of five explanatory models. Clinical Psychology Review, 19(3), 297-328.

16. Roszia, S. K., and Maxon, A. D. (2019). Seven core issues in adoption and permanency. Philadelphia, PA: Jessica Kingsley Publishers.

17. Stokes, L. D. and Poulsen, S. S. (2011). Narrative therapy for adoption issues in families, couples, and individuals: Rationale and approach. Journal of Family Psychotherapy, 25, 330-347.

18. Wade, J., and Alewine, E. (2018). An exploration of family challenges and service needs during the post-adoption period. Children and Youth Services Review, 91, 213-220.

19. Weistra, S., and Luke, N. (2017). Adoptive parents' experiences of social support and attitudes. Adoption and Fostering, 41(3), 228-241.

20. Workshops. (n.d.) Retrieved March 18, 2020 from Center for Child and Family Health's. 